\title{
Effects of Total Anthraquinones of Polygonum Cuspidatum on the Levels of Insulin, Leptin and C-Peptide in Serum in Diabete Rat Model
}

\author{
Ming BAI, Yan LI, Mingsan MIAO*, Ting WANG, Yuyin XU \\ Henan University of Traditional Chinese Medicine, Zhengzhou 450046, China
}

\begin{abstract}
To investigate the effect of total anthraquinone of Polygonum cuspidatum (TAPC) in diabete rats which were established by streptozotocin. Method: The diabete rat model was established by injected with streptozotocin, and then randomized into 5 groups. Which were fed with high dosage TAPC group, medium dosage TAPC group, low dosage TAPC group $\left(400 \mathrm{mg} \bullet \mathrm{kg}^{-1}, 200 \mathrm{mg}^{\bullet} \mathrm{kg}^{-1}, 100 \mathrm{mg} \cdot \mathrm{kg}^{-1}\right)$, metformin group $\left(208 \mathrm{mg} \bullet \mathrm{kg}^{-1}\right)$ and model group. Control group was set up with healthy normal rats. All the rats were administrated once a day for 30 days. Blood glucose (BG) in the limosis were measured on the 20,30 day and then rats were sacrificed. The levels of insulin, insulin antibody, leptin, C-peptide in orbital blood were measured. Results: Diabetic model rats successfully. Compared with model group, The high dosage TAPC group can significantly reduce the level of blood suga on 30 day $(P<0.01)$, Can significantly reduce the level of blood suga on 20 day $(P<0.05)$; The medium dosage TAPC group significantly reduce the level of blood suga on 20, 30 day $(P<0.01)$; The medium and low dosage TAPC group significantly increased the level of insulin $(P<0.01)$; Each dosage TAPC group can significantly reduce the level of $\mathrm{C}$-peptide $(P<0.01)$. Conclusion: TAPC has good hypoglycemic effect on the diabete rats established by injection of streptozotocin KEYWORD: Total anthraquinone of Polygonum cuspidatum (TAPC); Diabete model; Streptozotocin; Insulin antibody; Leptin; C-peptide.
\end{abstract}

\section{INTRODUCTION}

Polygonum cuspidatum, also known as "Kowloon root", "Yin Yang Lin", tepid, bitter, liver, gall bladder, lung, as Polygonum cuspidatum Sieb.et Zucc of dried roots and rhizomes, which as the medicine first appeared in "Thunder Gun Sunburn"[1]. The main effect is expelling wind and dampness, stasis and pain, cough and phlegm. Modern research shows that Polygonum cuspidatum contains anthraquinone compounds, flavonoids, water-soluble polysaccharides, tannins and trace elements and other components[2]. The total anthraquinone of Polygonum cuspidatum, including emodin ether, stilbene compound, which also contains tannin and polysaccharide[3].Modern pharmacological study have found that aqueous extract of Polygonum cuspidatum $\alpha$ - glucosidase was significantly inhibited[4]; Polygonum cuspidatum had been reported that anthraquinone compounds for hypoglycemic active ingredients[5]; Another report the tannins of Polygonum cuspidatum has good hypoglycemic effect on the alloxan diabetic mice[6]. Polygonum cuspidatum as promoting blood circulation to remove blood stasis hypoglycemic drugs in folk had application [7]. In order to investigate the characteristics of Polygonum cuspidatum and effect on the treatment of diabetes, the experimental to investigate the effect of total anthraquinone of Polygonum cuspidatum on streptozotocin-induced diabetic rats model.

\section{MATERIALS}

\subsection{Animals}

Wistar rats, male, whose weight were $180-200$ g, were supplied by the Experimental Animal Center of Hebei Province (Animal permit number: 701022).

\subsection{The experimental reagents and drugs}

The TAPC, provided by the Henan University of Traditional Chinese Medicine chemistry department, content more than 50\%; Metformin Hydrochloride Tablets was from Shanghai Pharmaceutical Group Co.,Ltd. Xinyi Pharmaceutical Factory; Streptozotocin (STZ) was from sigma company; Physiological saline, zhengzhou yonghe pharmaceutical co., LTD; Citric acid (AR) was from 
Hubei Pharmaceutical company Bose station; Glucose kit was from Zhejiang East Ou Biological Engineering Co., Ltd.Insulin radiation immunoassay kit, c-peptide radiation immunoassay kit and insulin antibody radiation immunoassay kit were all from Beijing Kemei Doya Biological Technology Co., Ltd.

\subsection{The experimental instrument}

UV-2000 UV-visible spectrophotometer, UNICO (Shanghai) Instrument Co., Ltd; Constant temperature water bath, Beijing Guangming Instrument Factory; FA (N) /JA (N) series electronic balance, Shanghai Minqiao Precision Instrument Co., Ltd; Adjustable liquid shifter, Shanghai Leibo Analysis Instrument Co., Ltd.

\section{METHODS}

$100 \mathrm{Wistar}$ male rats. After fasting 12h,Random take 90 rat formulated at $\mathrm{pH} 4.2$ ) after fasting $12 \mathrm{~h}$ and the remaining as the blank group, Tail vein injection of an equal volume of citrate buffer[8]. On the tenth day, tail blood test blood sugars, select 50 rats which $\mathrm{BG}>11.1 \mathrm{mmol} \cdot \mathrm{L}^{-1}$, and obviously drinking, eating, urining, then were fed with high, medium and low dosage TAPC suspension $\left(400 \mathrm{mg} \cdot \mathrm{kg}^{-1}, 200 \mathrm{mg} \bullet \mathrm{kg}^{-1}\right.$, $100 \mathrm{mg}^{\prime} \mathrm{kg}^{-1}, \quad 20 \mathrm{mg} \cdot \mathrm{ml}^{-1}, \quad 10 \mathrm{mg} \cdot \mathrm{ml}^{-1}, \quad 5 \mathrm{mg}^{-1} \mathrm{ml}^{-1}$, $\left.2 \mathrm{ml} \cdot 100 \mathrm{~g}^{-1}\right)$, metformin suspension $\left(208 \mathrm{mg}^{\circ} \mathrm{kg}^{-1}\right.$, $\left.20.8 \mathrm{mg} \cdot \mathrm{ml}^{-1}, 2 \mathrm{ml} \cdot 100 \mathrm{~g}^{-1}\right)$, model group and blank control group were given the same volume physiological saline. Once a day, treated for 30 days.
When the tenth, twentieth, thirtieth day, measured the blood glucose of 2 hours after administration (fasting12h), tail blood and measured the level of BG. In the last fasting blood sugar test one day before $12 \mathrm{~h}$, drenching $2 \mathrm{~h}$ after blood serum was separated, according to kit instructions were Determination of insulin, C- peptide and leptin levels.

\section{STATISTICAL ANALYSIS}

Data analysis used SPSS 13.0 for windows for statistical treatment. The differences of measurement data between groups were analyzed using ANOVA, ranked data used Ridit test.

\section{RESULTS}

\subsection{Impact on blood sugar in the STZ rat model}

From table 1, compared with the blank group, the level of BG in tenth, twentieth, thirtieth day was significantly increased $(P<0.01)$, shows that the model successfully. Compared with the model group, in twentieth, thirtieth day, the medium dosage TAPC group and metformin group could remarkably degrade the level of BG $(P<0.01)$; In thirtieth day, the high dosage TAPC group could remarkably degrade the level of BG $(P<0.01)$; In twentieth day, the high, dosage TAPC group could obviously degrade the level of BG $(P<0.05)$; Low dosage TAPC group has a tendency to lower the level of BG.

Table 1. Effect of TAPC on blood sugar in the STZ rat model ( $\bar{x} \pm s, n=10$ )

\begin{tabular}{|c|c|c|c|c|c|}
\hline \multirow{2}{*}{ Group } & \multirow{2}{*}{ Dose $(\mathrm{mg} \cdot \mathrm{kg}-1)$} & \multicolumn{4}{|c|}{ The level of BG(mmol $\left.\cdot \mathrm{L}^{-1}\right)$} \\
\cline { 3 - 6 } & & Began to glucose & $10 \mathrm{~d}$ & $20 \mathrm{~d}$ & $30 \mathrm{~d}$ \\
\hline Blank group & & $4.824 \pm 0.858^{* *}$ & $4.982 \pm 0.766^{* *}$ & $4.735 \pm 1.094^{* *}$ & $4.862 \pm 0.718^{* *}$ \\
\hline Model group & & $15.473 \pm 2.600$ & $17.755 \pm 2.492$ & $18.464 \pm 2.356$ & $19.766 \pm 2.754$ \\
\hline Metformin group & 208 & $15.870 \pm 2.936$ & $15.663 \pm 2.090$ & $13.585 \pm 2.061 * *$ & $11.584 \pm 1.922 * *$ \\
\hline High dosage TAPC group & 400 & $15.660 \pm 2.805$ & $16.904 \pm 3.266$ & $15.754 \pm 2.653 *$ & $13.896 \pm 2.529 * *$ \\
\hline Medium dosage TAPC group & 200 & $16.233 \pm 3.019$ & $16.471 \pm 1.860$ & $14.487 \pm 2.889 * *$ & $12.612 \pm 2.287 * *$ \\
\hline Low dosage TAPC group & 100 & $15.910 \pm 2.811$ & $17.625 \pm 2.388$ & $17.901 \pm 2.924$ & $17.842 \pm 3.327$ \\
\hline
\end{tabular}

Note: Compared with the model group, $* \mathrm{P}<0.05$, $* * P<0.01$

\subsection{Impact on Serum insulin, $C$ - peptide and leptin levels in the STZ rat model}

From table 2, Compared with the control group, the model group could significantly degrade levels of insulin and C-peptide levels $(P<0.01)$, significantly increase leptin levels $(P<0.01)$, It suggests that the model copied successfully. Compared with the model group, medium dosage TAPC group and metformin group could significantly increase serum insulin levels $(P<0.01)$, high dosage TAPC group could significantly increase serum insulin levels $(P<0.05)$; Medium and low dosage TAPC group can significantly increase serum $C$ - peptide levels $(P<0.01)$; High, medium and low dosage TAPC group can significantly degrade serum leptin levels $(P<0.01)$. 
Table 2. Effect of TAPC on Serum insulin, C-peptide and leptin levels in the STZ rat model $(\bar{x} \pm s, n=10)$

\begin{tabular}{|c|c|c|c|c|}
\hline Group & Dose $\left(\mathrm{mg} \cdot \mathrm{kg}^{-1}\right)$ & Serum insulin $\left(\times 10-3 \mu \mathrm{IU} \cdot \mathrm{ml}^{-1}\right)$ & C-peptide $\left(\mathrm{ng} \cdot \mathrm{ml}^{-1}\right)$ & $\mathrm{Leptin}\left(\mathrm{ng} \cdot \mathrm{ml}^{-1}\right)$ \\
\hline Blank group & & $47.280 \pm 8.471 * *$ & $0.109 \pm 0.034 * *$ & $0.645 \pm 0.180^{* *}$ \\
\hline Model group & & $26.850 \pm 4.809$ & $0.043 \pm 0.016$ & $1.517 \pm 0.346$ \\
\hline Metformin group & 208 & $37.320 \pm 6.064 * *$ & $0.061 \pm 0.017$ & $0.849 \pm 0.469 * *$ \\
\hline High dosage TAPC group & 400 & $33.129 \pm 5.393 *$ & $0.054 \pm 0.016$ & $0.970 \pm 0.430^{* *}$ \\
\hline Medium dosage TAPC group & 200 & $36.887 \pm 5.077 * *$ & $0.101 \pm 0.021^{* *}$ & $0.630 \pm 0.235^{* *}$ \\
\hline Low dosage TAPC group & 100 & $29.927 \pm 5.884$ & $0.074 \pm 0.017 * *$ & $0.840 \pm 0.273^{* *}$ \\
\hline
\end{tabular}

Note: Compared with the model group, ${ }^{*} \mathrm{P}<0.05, * * P<0.01$

\section{DISCUSSION}

Diabetes in traditional Chinese medicine belong to the category of "excessive", and obviously drinking, eating, urining, thin body, or turbid urine, urine has a sweet taste; Modern medicine that diabetes is a chronic metabolic disease caused by a variety of causes, its basic pathology for the absolute or relative lack of insulin secretion and peripheral tissue is not sensitive to insulin, or cause is given priority to with sugar metabolic disorders, including fat and protein metabolism disorder of a systemic disease[9]. The experimental model by intravenous injection of STZ diabetic model making[10], because of its damaging effects on islet $\beta$ cell in experimental animals is highly selective, and its mechanism induced diabetic animal model is first islet $\beta$ cell in specific sites of DNA base alkylation further action ribosome synthesis in ADP enzyme and thus directly damage pancreatic $\beta$ cell by $\mathrm{NO}$ and $\mathrm{O} 2-\cdot$ radicals two paths, making the islet $\beta$ cell necrosis, insufficient insulin secretion, which can be successfully established diabetes model that is closer to human diabetes, has been widely used. Insulin, serum, C-peptide is an important indicator of insulin secretory function of cells; Leptin is a hormone gene from obesity produced, and insulin resistance are closely related [11]. Serum insulin, C-peptide is an important index reflecting cell secretion of insulin. Leptin is obese gene (OB) control coding, a cycle of endocrine hormone secreted by fat cells, which is one of the symbols of the direct effect of obesity and its main functions of the central nervous system leptin receptors, inhibiting the synthesis of neuropeptide $Y$, then to reduce appetite, reduce the intake of energy, and the relationship between leptin and insulin resistance is closely; As there is no cross reaction c-peptide and insulin antibodies, are not affected by the interference of insulin antibodies, its value can more directly, more objectively and more accurately reflects the function of islet beta cells [12] .

This study shows that the TAPC can significantly degrade blood glucose in the STZ rat model, there was no significant difference between its hypoglycemic effect of metformin; and in hypoglycemic while significantly elevated levels of serum insulin and $\mathrm{C}$ - peptide levels significantly lower leptin levels which elevated the role of $\mathrm{C}$ peptide levels significantly better than metformin group; but the regulation of insulin antibodies was not obvious. The result suggest that the TAPC in Polygonum cuspidatum has good curative effect on streptozotocin induced diabetic rat model, can stimulate the secretion of islet $\mathrm{C}$ - peptide and insulin, improve the symptoms of diabetes. This experiment is used in the treatment of diabetes Polygonum cuspidatum provided data support and support, but also for the further development and utilization of Polygonum cuspidatum foundation.

\section{ACKNOWLEDGEMENTS}

The research work is supported by the authentic ingredients deep processing of Henan Engineering Laboratory (2012-1431-10); Scientific and Technological Innovation Team (2012IRTSTHN011); Zhengzhou Municipal Science and Technology Innovation Team program (131PCXTD612); Efficacy rating Engineering Technology Center Education Science and Technology (2012) 78-12); The Collaborative Innovation Center for the creation of new Traditional Chinese medicine and genuine regional drug deep processing in Henan University of Traditional Chinese Medicine (2012) 188-2.

\section{REFERENCES}

[1] Huang HL. Pharmacological Progress of HuZhang. Western Journal of Traditional Chinese Medicine, 2012, 25(4): 100-103.

[2] Li P. Quality control of giant knotweed rhizome and its chemical constituents. Qilu Pharmaceutical Affairs, 2011, 30(4): 232-238.

[3] Fan HT, Ding SL, Lin HS. Research Progress on pharmacological activities of Polygonum cuspidatum Sieb. Chinese journal of traditional Chinese medicine (TCM), 2013, 38(15): 2545-2548. 
[4] Lin YH, Li J. Screening of $\alpha$-Glucosidase Inhibitor from Polygonum cuspidatum Siebet Zucc. Journal of Anhui Agricultural Sciences, 2010 38(17):8986-8988.

[5] Wang H, Yang ZG, Yan SX, et al. The influence of lipid metabolism and blood rheology of total anthraquinone of Polygonum cuspidatum on early diabetic nephropathy rat blood stasis model. Chinese Journal of Experimental Traditional Medical Formulae, 2010, 16(16):155-159.

[6] Qin Y. Summary of clinical application of metformin. Continuing Medical Education, 2012, 26(3): 46-48.

[7] Xu XZ. The Researching Progress of Ttreating Diabetic Nephropathy by Blood-activating and Statis-dissolving Chinese. Asia-Pacific Traditional Medicine, 2014, 10(3): 49-52.

[8] Miao MS, Li CR, Chen PY. Effects of Total Flavonoids of Sophorae Flos on the Levels of Insulin, Leptin and C-
Peptide in Serum in Diabete Rat Model. Chin J Mod Appl Pharm, 2011, 28(10):896-898.

[9] Fan W, Liu Q, Tian HQ. New progress in the research and development of anti-diabetic drugs. Chinese Journal of New Drugs, 2013, 22(21):2503-2509.

[10] Miao MS, Cheng BL, Jiang N. Effect of Sophore Japonica Total Flavonoids on Models of Hyperglycemia and Diabetes Model, Applied Mechanics and Materiais, 2014;664(1):388.

[11] Chen YX, Wei JB. Researching advance of type 2 diabetes complications rat models induced by streptozotocin. Chinese Journal of Comparative Medicine, 2013, 23(3): 63-66.

[12] Miao MS, Cheng BL, Jiang N, Jiang MQ. Qumai Total Flavonovids Induced Diabetic Mice of Streptozotocin. Mechatronics and Mechanical Engineering, Applied Mechanics and Materiais, 2014, 664(1):397. 